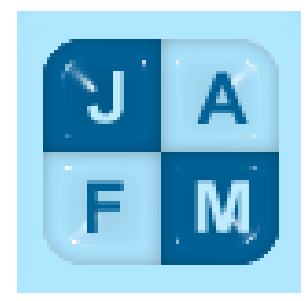

\title{
Experimental Investigations on Oscillatory Couette Taylor Flow Wall Shear Stress Behavior using Electrochemical Technique: Low Modulation Effect
}

\author{
E. Berrich Betouche ${ }^{1}$, F. Aloui ${ }^{2}$ and J. Legrand ${ }^{3}$ \\ ${ }^{I}$ GEPEA - UMR CNRS 6144, Ecole des Mines de Nantes, Energetic Systems and Environment Department, \\ 4 Alfred KASTLER Street-BP20722 Nantes, France \\ ${ }^{2}$ Université de Valenciennes et du Hainaut-Cambrésis (UVHC), LAMIH CNRS UMR 8201, Département de \\ Mécanique, Campus Mont Houy, F-59313 Valenciennes Cedex 9, France \\ ${ }^{3}$ LUNAM Université, Université de Nantes, CNRS, GEPEA UMR6144, CRTT, BP 406, \\ 37 Boulevard de l'Université - 44602 Saint-Nazaire Cedex, France \\ E-mail: Fethi.Aloui@univ-valenciennes.fr
}

(Received October 20, 2015; accepted December 10, 2015)

\begin{abstract}
In the simplest and original case of study of the Taylor-Couette TC problems, the fluid is contained between a fixed outer cylinder and a concentric inner cylinder which rotates at constant angular velocity. Much of the works done has been concerned on steady rotating cylinder(s) i.e. rotating cylinders with constant velocity and the various transitions that take place as the cylinder(s) velocity (ies) is (are) steadily increased. On this work, we concentrated our attention in the case in which the inner cylinder velocity is not constant, but oscillates harmonically (in time) clockwise and counter-clockwise while the outer cylinder is maintained fixed. Our aim is to attempt to answer the question if the modulation makes the flow more or less stable with respect to the vortices apparition than in the steady case and if there are any reversing or non reversing flows apparition. If the modulation amplitude is large enough to destabilize the circular Couette flow, two classes of axisymmetric Taylor vortex flow are possible: reversing Taylor Vortex Flow (RTVF) and Non-Reversing Taylor Vortex Flow (NRTVF). Our work presents an experimental investigation of the effect of oscillatory Couette-Taylor flow on the instantaneous and local mass transfer and wall shear rates evolutions, i.e. the impact of vortices at wall; and the detection of any RTVF and/or NRTVF apparition. The vortices may manifest themselves by the presence of timeoscillations of mass transfer and wall shear rates; this generally corresponds to an instability apparition even for steady rotating cylinder. On laminar CT flow, the time-evolution of wall shear rate is linear. It can be presented as a linear function of the angular velocity. For a mean Taylor number corresponding to a laminar Couette flow, a modulation frequency $F=0.1 \mathrm{~Hz}$ and an amplitude respectively $\beta=0.53$ and $\beta=1.08$ are sufficient to destabilize the laminar CT flow, Taylor vortices appear. Comparing to a steady rotational velocity case, oscillatory flow accelerates the instability apparition, i.e. the mean critical Taylor number corresponds to the transition is smaller than that of the steady rotational case. The vortices direction can be deduced from the sign of the instantaneous wall shear rate time evolution.
\end{abstract}

Key Words: Couette-Taylor(CT); Electrochemical technique; Experiments; Transition; Instability; Taylor number.

NOMENCLATURE

$\begin{array}{ll}\text { a } & \text { inner cylinder radius } \\ \text { A } & \text { surface of the probe } \\ \text { C } & \text { concentration } \\ \text { D } & \text { coefficient of diffusion } \\ \text { F } & \text { frequency of oscillation }\end{array}$

$\Omega \quad$ angular velocity of the rotating plate

$u \quad$ kinetic viscosity

$\delta \quad \delta=\mathrm{R}_{2}-\mathrm{R}_{1}$

$\eta \quad$ radial ratio

$\Gamma \quad$ aspect ratio 
E. B. Betouche et al. /JAFM, Vol. 9, Special Issue 1, pp. 147-154, 2016.

$\begin{array}{ll}\mathrm{H} & \text { height of the CTS } \\ \mathrm{I} & \text { current } \\ \mathrm{l} & \text { probe length } \\ \mathrm{Pe} & \text { Péclet number } \\ \mathrm{R} & \text { radius } \\ \mathrm{Re} & \text { Reynolds number } \\ \mathrm{S} & \text { wall shear stress } \\ \mathrm{Sh} & \text { sherwood number } \\ \mathrm{t} & \text { time } \\ \mathrm{Ta} & \text { Taylor number } \\ \mathrm{u} & \text { velocity } \\ \mathrm{x}, \mathrm{z} & \text { coordinates } \\ \mathrm{Greek} & \text { symbols } \\ \beta & \text { amplitude of the oscillation }\end{array}$

\section{INTRODUCTION}

Couette-Taylor (CT) flows are frequently reencountered on engineering processes (such on rotorstator systems) and medical applications. It is widely used for the depleted uranium enrichment, as a membrane or filter...Most of the studies were interested to the Couette-Taylor simple case where the fluid is contained between a fixed outer cylinder and a concentric inner cylinder which rotates at constant angular velocity. We called this case "the steady rotating cylinder(s)"case such as the recent work of Abcha et al. (2013)who studied spiral vortex flow in counter-rotating Couette-Taylor system (SCT) with a large aspect ratio and an intermediate gap using the laser technique "Particle Image Velocimetry" (PIV) which is a global technique for velocity field determination. However, few studies focus on flow dynamic perturbation effects such as axial and/or radial flow imposed to rotating flow (Tilton et al., 2010; Martinand et al., 2009) or heat transfer combined to flow dynamics (Kedia et al., 1999; Gilchrist et al., 2005). Less works were interested to mass transfer combined to rotating flow dynamics and wall interaction on Couette Taylor system such as Berrich et al., 2012 (a); 2012 (b), 2013 (a); Kristiawan et al., (2011)... However, Kristiawan et al., (2011) work has concerned the determination of wall shear rate from mass transfer for steady rotating cylinder. While our works focused on steady rotating flow combined to axial flow (Berrich et al., 2012 (a) and (b)) and oscillating flows (Berrich et al., 2013 (a)) effects on mass transfer and wall shear rate.

The occurrence of modulated flows in nature and in technological applications such as the stability of periodic blood flow in the aorta (Skalak et al., 1989), the alternation of heating and cooling of planetary atmospheres, encourages researchers to study parametric excitation effect on hydrodynamic (Feugaing et al., 2011). Donnelly et al., (1962) enumerated some theoretical (Hall, 1975; Hall, 1983; Riley and Laurence, 1976), experimental (Barenghi and Jones, 1989; Barenghi, 1991; Ganske et al., 1994) and numerical (Walsh and Donnelly, 1988) studies.

$\begin{array}{ll}\begin{array}{l}\text { Superscripts } \\ *\end{array} & \text { dimensionless } \\ & \text { temporal average } \\ \overline{\text { Subscripts }} \\ \text { a } \quad \text { inertia effect } \\ \text { ax } & \text { axial } \\ \mathrm{c} & \text { critical } \\ \text { cent } & \text { centrifugal force } \\ \mathrm{i} & \text { inner } \\ \text { q.1. } & \text { quasi-linear } \\ \text { Lev } & \text { lévêque approach } \\ \text { Sob } & \text { Sobolik et al. approach } \\ \text { Osc } & \text { oscillatory } \\ \text { Max } & \text { Maximum }\end{array}$

These works illustrate that the oscillation motion destabilizes the flow but reveal a disagreement on the fact that a low frequency modulation produces a large or small destabilizing effect. However, few experimental studies have investigated the effect of modulation on mass transfer and wall shear rate. On this work, we concentrated our attention to the case in which the inner cylinder velocity is not constant, but oscillates harmonically (in time) clockwise and counter-clockwise while the outer cylinder is maintained fixed. Our aim is to attempt to answer the questions if the modulation makes the flow less stable with respect to the vortices apparition than in the steady rotating case and if there are any Reversing Taylor Vortex Flow (RTVF) or/and Non-Reversing Taylor Vortex Flow (NRTVF).

If the modulation amplitude is large enough to destabilise the circular Couette flow, two classes of axisymmetric Taylor vortex flow are possible: Reversing Taylor Vortex Flow (RTVF) and NonReversing Taylor Vortex Flow (NRTVF). For Reversing Flow, the inner cylinder is rotating anticlockwise for the first part of the cycle; the vortices respond by rotating in the same cylinder motion direction. For the second part of the cycle, the inner cylinder rotates in the opposite direction, the vortices respond by changing their rotation direction. For NonReversing Flow, the inner cylinder and the vortices rotate in the same direction. However, when the inner cylinder reverses its rotation direction, the vortices don't follow the new cylinder direction i.e. they continue to rotate in the same direction as in the first half-cycle. Youd et al. (2003) studied numerically the two flow types. They demonstrated that for relatively high oscillation frequencies, the Taylor vortices direction does not change every half-cycle, i.e. presence of NRTVF. Youd et al. (2005) proved that, for Couette-Taylor system characterized by a radial ratio $\eta=R_{\text {int }} / R_{\text {out }}=0.75$, there was a transition to an axisymmetric time-modulated flow, i.e. the NonReversing Taylor Vortex Flow (NRTVF) in which Taylor vortices rotation direction is independent on the inner cylinder rotation direction. They illustrated that if the oscillation amplitude is large enough, the resulting time-dependent Taylor vortex can rotate in a 
E. B. Betouche et al. /JAFM, Vol. 9, Special Issue 1, pp. 147-154, 2016.

direction independent on the direction of the azimuthal motion which drives it. Taylor vortex pairs always rotate in the same direction, despite the inner cylinder drives the flow in the opposite direction. According to Youd et al., (2005) work, NRTVF takes place at sufficiently high modulation frequencies for which there is not enough time for toroïdal motion to vanish to small values. It can be caused by linear instability or finite-amplitude effects (Youd et al. 2003). It may be affected by the weak Ekman circulation (absent in the calculation of Youd et al. (2003) which is induced by the fixed top and bottom ends of the Taylor-Couette System. Carmi and Tustaniwskyj (1981) numerically studied oscillatory Couette flow. They did not detect the existence of NRTVF. Their approach was based on studying the effects of infinitesimal perturbations over a cycle, i.e. the Floquet theory. More details about the theory are available on (Youd et al., 2003; Carmi and Tustaniwskyj, 1981; Barenghi and Jones, 1989). It is possible that NRTVF is due to finite-amplitude effects, whereas in RTVF, the radial velocity becomes negative for a part of the cycle. However, NRTVF could be due to a linear instability too according to Lopez and Marques (2002) Floquet analysis.

Our work presents an experimental investigation of oscillatory Couette-Taylor flow behaviour, i.e. modulation effect on the apparition of RTVF and NRTVF by analyzing the instantaneous and local mass transfer and wall shear rate evolutions, i.e. the impact of vortices at wall.

\section{EXPERIMENTAL INSTALLATION}

The experimental installation (Fig. 1) is composed of an inner cylinder with a radius $R_{1}=97 \pm 0.2 \mathrm{~mm}$ and an outer cylinder with a radius $\mathrm{R}_{2}=100 \pm 0.2 \mathrm{~mm}$. The radius ratio is $\eta=0.97$. The aspect ratio is $\Gamma=150$. The whole apparatus is made in Plexiglas. The inner cylinder was driven by a $\alpha$-Step motor (marketed by the Omeroncompany). The electrical motor imposed to the inner cylinder a maximum speed of $120 \mathrm{rpm}$. It was supplied by a signal generator. The revolutions were controlled by a speed controller. The engine assures to impose sinusoidal motion to the inner cylinder.

\section{POLAROGRAPHY TECHNIQUE}

Polarography (electrochemical) technique, known as the electro-diffusion method, has been used. This requires the use of Electro-Diffusion (ED) probe which delivers the Limiting Diffusion current while it is polarized by a well polarization voltage. The local mass transfer rate is determined from the current. Then, wall shear rate is determined from mass transfer signals. In addition, a series of platinum probes have been mounted flush to the inner surface of the outer cylinder of the Couette-Taylor System (CTS). ED results are presented for the probe $\mathrm{G}$ situated at the mid-height of the CTS (Fig. 1).

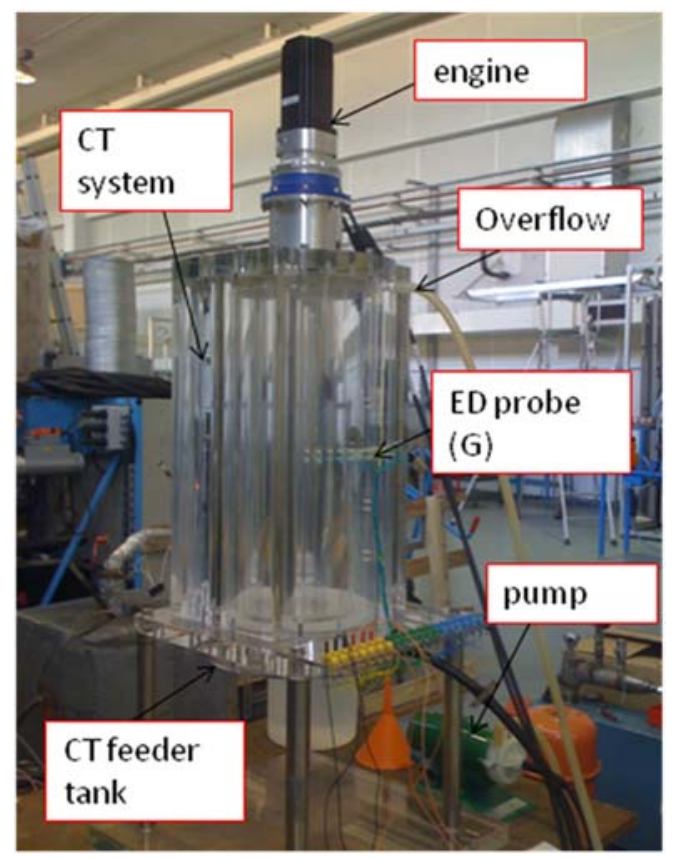

Fig. 1. Experimental installation.

In our tests, a ferri-ferrocyanure of potassium is used as electrochemical solution with a concentration of 25 $\mathrm{mol} / \mathrm{m}^{3}$. An access of the sulfate of potassium $\mathrm{K}_{2} \mathrm{SO}_{4}$ $\left(130 \mathrm{~mol} / \mathrm{m}^{3}\right)$ is added as supporting electrolyte and $40 \%$ of Glycerin to relay the instability apparition (viscosity effect).

\section{STEADY TAYLOR VORTEX FLOW CHARACTERIZATION}

To attempt to answer the question if the modulation makes the flow more or less stable with respect to the vortices apparition than in the steady rotational case, we begin by characterizing the flow for the case where the inner cylinder rotates at constant angular velocity i.e. steady rotational case.

The Taylor number is generally used to characterize modes transition. It describes the competition between the viscous dissipation and the centrifugal force. It is defined as:

$$
T a=\frac{\tau_{v}}{\tau_{\text {cent }}}=\frac{\Omega R_{\text {int }} \delta}{v} \sqrt{\frac{\delta}{R_{\text {int }}}}=R e_{i} \sqrt{\frac{\delta}{R_{\text {int }}}}
$$

where the Reynolds number describes the competition between the viscous dissipation and the inertia effect:

$$
R e=\frac{\tau_{v}}{\tau_{a}}=\frac{\Omega R \delta}{v}
$$

The time-evolution of the Taylor number is presented 
on Fig. 2. It shows that the Taylor number is constant and that the average Taylor number is equal to $8 \pm 0.4$. For this Taylor number, we studied the time-evolution of mass transfer and wall shear rates. This allows flow instability detection because the vortices manifest themselves by the apparition of variations on mass transfer and wall shear rates evolutions.

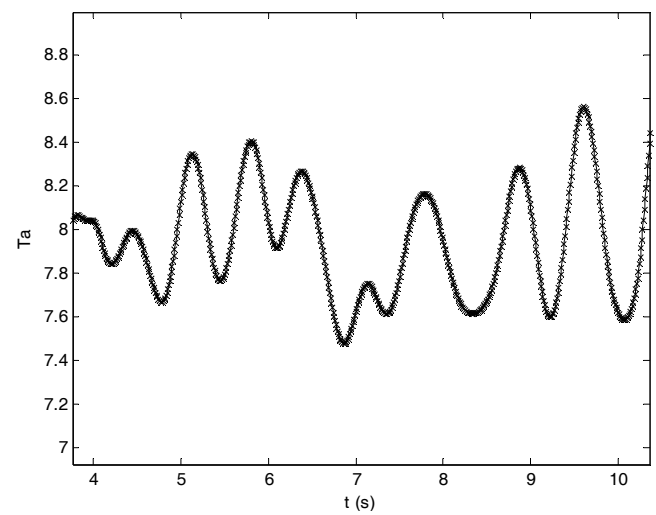

Fig. 2. Evolution of the Taylor number for steady rotational flow case.

The dimensionless mass transfer rate is almost constant (Fig. 3). The average value is equal to $1 \pm 0.02$. The flow regime is thus laminar. It corresponds to Couette flow. The time-evolution of

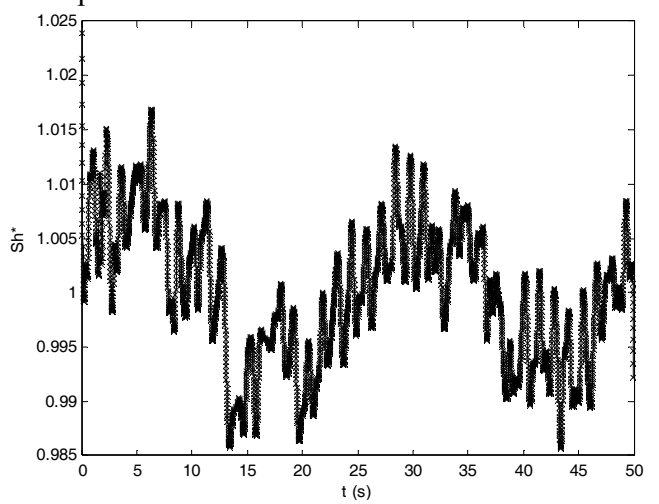

Fig. 3. Evolution of dimensionless mass-transfer rate for $T \mathbf{T}_{\mathrm{m}}=\mathbf{8}$.

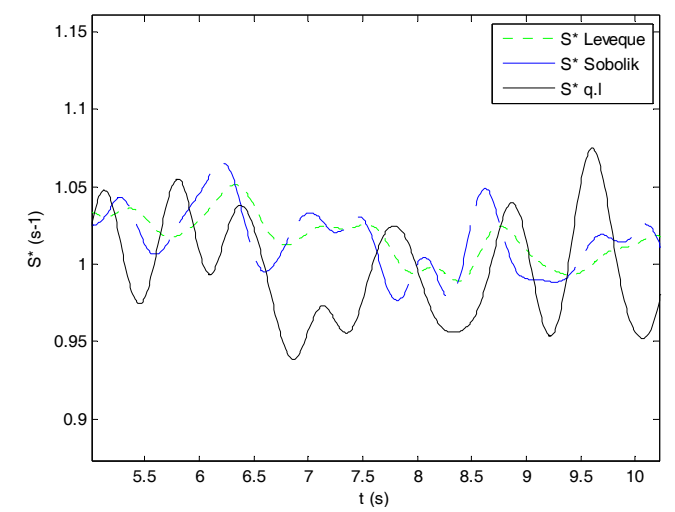

Fig. 4. Evolution of dimensionless wall shear stress for $T a_{m}=8$. dimensionless wall shear rate for $\mathrm{Ta}=8$ is presented on Fig. 4. The dimensionless wall shear rate remains constant. The average value is equal to $1 \pm 0.05$. The flow is thus stable and no vortices appear. On laminar CT flow, the time-evolution of wall shear rate is linear. It may be presented as a linear function of the angular velocity.

For the same average inner cylinder velocity, we studied the effect of oscillations on flow.

\section{MODULATION EFFECT ON RTVF AND/OR NRTVF}

\subsection{Protocols and Taylor number evolutions}

As the RTVF and the NRTVF strongly depend on the inner cylinder rotation direction, we studied two principle protocols:

- CASE A: The first one corresponds to non-stop advancing inner cylinder i.e. the inner cylinder always advances while oscillating.

- CASE B: The second protocol corresponds to advancing - stopped inner cylinder.

The time-evolutions of the Taylor number are shown respectively on Fig. 5 (case A) and Fig. 6 (case B).As we can see, for the different oscillatory cases, the Taylor number presents a sinusoidal evolution characterizing the oscillatory flow presence. The

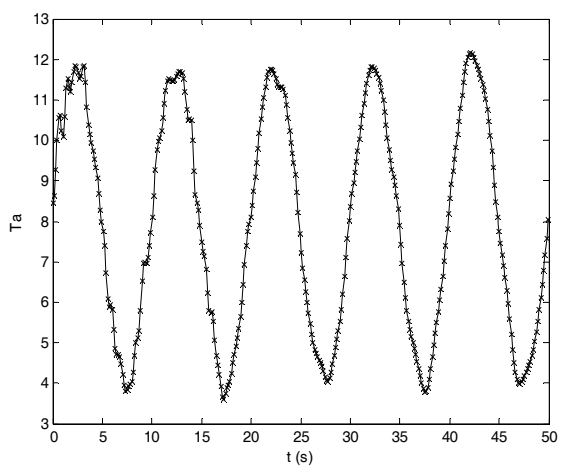

Fig. 5. Evolution of the Taylor number for $F=100$ $\mathrm{mHz}$ and $\boldsymbol{\beta}=\mathbf{0 . 5 3}$ (case $\mathrm{A})$.

average Taylor number is equal to 8 which correspond to Taylor number for the steady case studied on the first section. On case A, the modulation amplitude $\beta$ is strictly lesser than the unit and the Taylor number remains strictly positive. The Taylor number varies between 12 and 4 . On case $B, \beta$ is equals to the unit. The Taylor number time-evolution presents a zero Taylor number when the inner cylinder is stopped and strictly positive values when it advances. The cylinder oscillates between $\mathrm{Ta}=0$ and $\mathrm{Ta}=16$.

The average Taylor number, for all cases; is equals to 8 which correspond to Taylor number for the steady laminar Couette flow case studied on the first section. 
E. B. Betouche et al. /JAFM, Vol. 9, Special Issue 1, pp. 147-154, 2016.

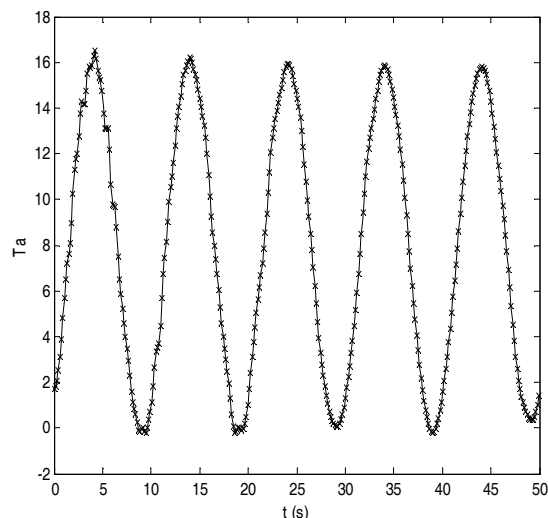

Fig. 6. Evolution of the Taylor number for $\mathrm{Ta}_{\mathrm{m}}=8$, $F=100 \mathrm{mHz}$ and $\beta=1.08$ (case $B$ ).

As we have proved that for Taylor number equal to 8 , the flow corresponds to laminar Couette Flow. The question now is to know if the oscillation disturbs the flow enough by accelerating the transition apparition and if there is any RTVF or NRTVF. We propose an answer by analyzing the mass transfer and the wall shear rates.

\subsection{Mass transfer rates analysis}

Mass transfer rates time-evolution i.e. the Sherwood number for the different oscillatory flow are presented respectively on Fig. 7 (case A), Fig. 8 (case B).

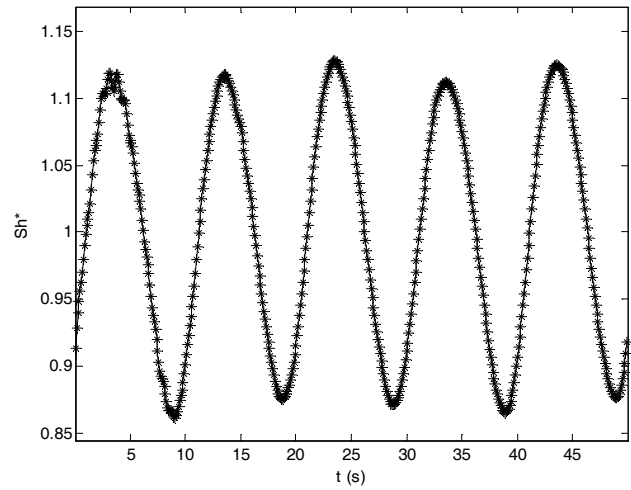

Fig. 7. Evolution of dimensionless experimental mass transfer

rate for $T a_{m}=8, F=100 \mathrm{mHz}$ and $\beta=0.53$.

The Sherwood number is defined by:

$$
S h=\frac{i l}{n F C_{0} A D}
$$

Where $i$ is the Limiting Diffusion current delivered by the Electro-Diffusion (ED) probe, $l$ the probe length, $n$ the ions number, $F$ the Faraday number, $C_{0}$ the initial concentration, $A$ the probe area and $D$ the coefficient of Diffusion.

The instantaneous and local mass transfer rates evolutions demonstrate the apparition and

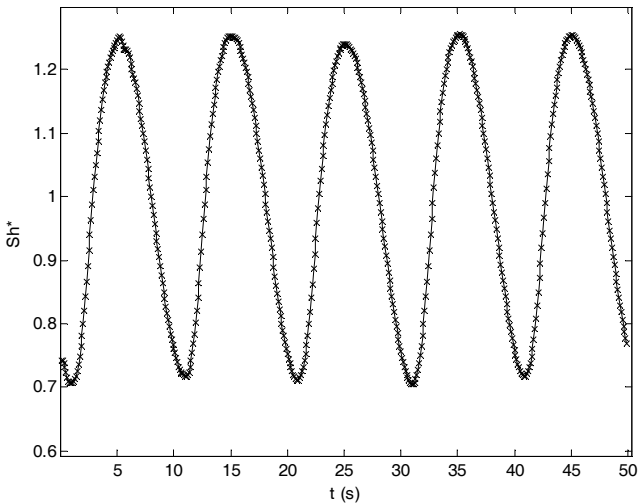

Fig. 8. Evolution of dimensionless experimental mass transferrate for $\mathrm{Tam}=8, \mathrm{~F}=100 \mathrm{mHz}$ and $\beta=1.08$.

development of Taylor vortices which manifest themselves by the presence of oscillations having constant amplitudes for $\beta=0.53$ (case A) and $\beta=$ 1.08; despite that the mean Taylor number corresponds to a steady laminar case i.e. Couette flow. So, we can deduce that oscillations accelerate the transition apparition and thus the critical mean Taylor number corresponding to the transition from laminar Couette flow to Wavy Vortex flow is lesser than that of the steady case. For $\beta=0.53$ and $\beta=1.08$, the mass transfer rate is no longer linear, it has a sinusoidal evolution. It follows the cylinder motion. It remains always positive.

\subsection{Wall shear rates analysis}

Basing on mass transfer evolutions, we have found that, if the oscillation amplitude is $0.53 \leq \beta \leq 1.08$, laminar Couette flow can be destabilized, Taylor vortices appear.

The wall shear rate was determined using three approaches: the "Lévêque solution" (1928), Sobolik et al. (1987) method which is a correction of the Lévêque (1928) solution and the inverse method (2006). The different approaches are well developed on our previous work (Berrich et al., 2013 b). The inverse method was experimentally validated in our previous work (Berrich et al., 2013 c). It gives satisfactory results even for reversing flows.

The experimental mass transfer signals were smoothed (filtered) before using it to determine the wall shear rate because the different approaches are very sensitive to noise

The temporal evolutions of wall shear rates, for relatively low modulation frequency $\mathrm{F}=100 \mathrm{mHz}$, are presented respectively for modulation amplitude $\beta=$ 0.53 and $\beta=1.08$ on Fig. 9 and Fig. 10.The vortices manifest themselves by the presence of timeoscillations on wall shear rate evolution. For low modulation frequency $(\mathrm{F}=0.1 \mathrm{~Hz})$, and for 
E. B. Betouche et al. /JAFM, Vol. 9, Special Issue 1, pp. 147-154, 2016.

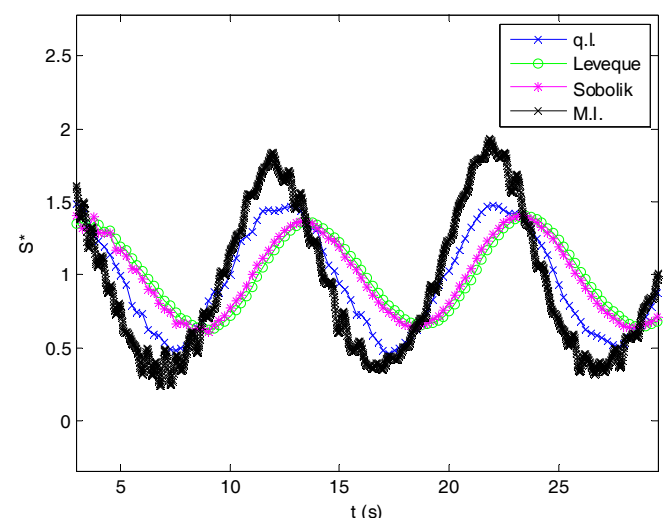

Fig. 9. Evolution of dimensionless wall shear rate for $\mathrm{Ta}_{\mathrm{m}}=8, \mathrm{~F}=100 \mathrm{mHz}$ and $\beta=0.53$.

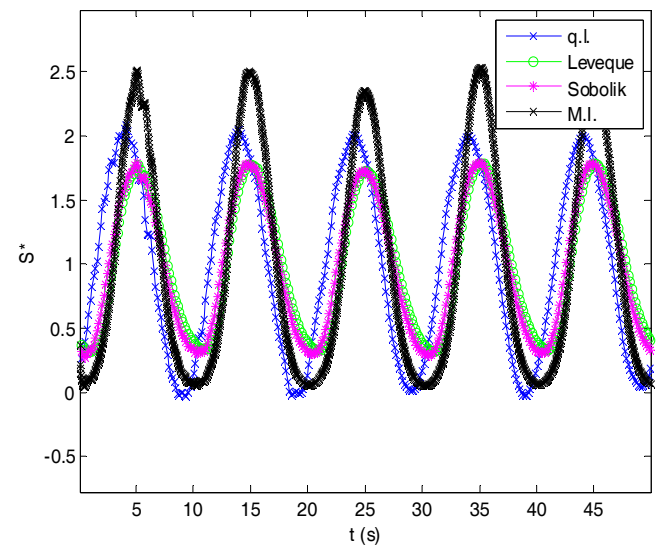

Fig. 10. Evolution of dimensionless wall shear rate for $T a_{m}=8, F=100 \mathrm{mHz}$ and $\beta=1.08$.

modulation amplitudes $\beta=0.53$ (i.e. nonstop advancing $\mathrm{CT}$ inner cylinder) and $\beta=1.08$ (i.e. advancing - stop advancing CT inner cylinder), wall shear rates still positive. We proved that if the oscillation amplitude is "small" enough, the resulting Taylor vortex rotates in a direction which do not depend on the direction of the azimuthal motion which drives it i.e. NRTVF.

\section{DETECTION CHARACTERIZATION \\ AND OSCILLATORY FLOWS USING FREQUENCY SPECTRUM}

The periodic signals usually used, whether low or high frequency, are rarely purely sinusoidal. They are in fact a mixture of sinusoidal signals whose frequencies are multiples of the fundamental frequency which is the lowest frequency. The multiple frequencies of the fundamental frequency are called harmonic. In general, any periodic function of frequency $f_{0}$ i.e. the fundamental frequency, can be decomposed into a sum of sinusoidal waves (harmonics) whose frequencies are integer multiples of $f_{0}\left(\begin{array}{llllll}2 & f_{0} & 3 & f_{0} & \ldots & \mathrm{N} f_{0}\end{array}\right)$. Its amplitudes and the



Fig. 11. Frequency spectrum $T a m=8, F=0.1 \mathrm{~Hz}$, $\boldsymbol{\beta}=\mathbf{0 . 5 3}$ (case A).

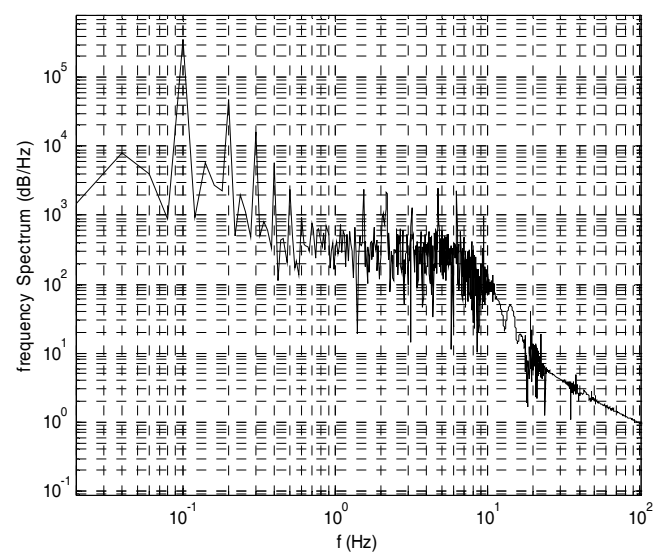

Fig. 12. Frequency spectrum $\mathrm{Ta}_{\mathrm{m}}=8, \mathrm{~F}=0.1 \mathrm{~Hz}$, $\boldsymbol{\beta}=\mathbf{1 . 0 8}$ (case B).

phases are the resulting of Fourier series decomposition.

Swinney and Zhang (2013) studied Taylor Vortex Flow dynamics in the case of rotating cylinders (steady rotational case). They defined the Reynolds number as $R=\left(2 \pi \cdot f_{c y l} \cdot a\right) \cdot d / v$; where $f_{c y l}$ is the cylinder frequency, $a$ is the inner cylinder radius, $b$ is the outer cylinder radius, $d=b-a$, and $v$ is the kinematic viscosity. They found that the transition from laminar flow to Taylor vortex flow, is an example of a "pitchfork" bifurcation, that is, a transition from one time-independent state to another with different symmetry. Modulated wavy vortex flow is characterized by two disproportionate characteristic frequencies. Wavy vortex state is characterized by spectra with a single fundamental frequency $f_{l}$ and its harmonics. In the wavy vortex state, an increase in $\mathrm{R}$ will lead to a transition where characterized by a frequency $f_{2}$. Their visual observations revealed that the Taylor vortex is modulated. The frequency $f_{2}$ corresponds to an azimuthal traveling wave similar to the wavy vortices; the angular speed of the modulated waves is about $0.44 f_{c y l}$. Proportionate and in proportionate frequencies in MWVF can never be 
E. B. Betouche et al. /JAFM, Vol. 9, Special Issue 1, pp. 147-154, 2016.

distinguished experimentally. A bifurcation from a time independent system to a periodic state is called a Hopf bifurcation.

Coughlin and Marcus (1992) studied the Modulated waves in TC flow. They resumed that in the experiments of Zhang and Swinney (1985) who used a Taylor-Couette system with radius ratio 0.883 , the flow state was determined by flow visualization, and spectra were taken from the time series of scattered light intensity. With this technique, there is no precise quantitative relationship between the amplitude of a spectral peak and the power of the corresponding mode (1985). Zhang and Swinney (1985) observed a quasi-periodic flow in which the spectral peak associated with the modulation, was frame independent and thus not associated with an azimuthally travelling mode.

We imposed a sinusoidal motion characterized by a fixed frequency and amplitude while conserving a mean Taylor number equals to $\mathrm{Ta}=8$ in order to compare the characteristics of the flow obtained to the steady rotational one, characterized also by $\mathrm{Ta}=8$, in term of vortices appearance and the flow regime obtained. What are the characteristic frequencies of these vortices?

The Figs 11 and 12 present a logarithmic presentation of the absolute values of Fourier Transform i.e. the frequency spectrum of mass transfer signals function of frequencies, for an imposed frequency oscillatory motion $f_{c y l}=F=0.1 \mathrm{~Hz}$.

For steady rotational flow characterized by $\mathrm{Ta}=8$, the flow is laminar i.e. Couette flow is characterized by vortices absence. Thus, the spectral peaks detected while imposing an oscillatory motion are the vortices

imprints i.e. the frequencies correspond to those of vortices which appear due to the modulation. There are commensurate (proportionate) and incommensurate frequencies (Table .1).

Table 1 Frequency spectrum

\begin{tabular}{|c|c|c|}
\hline $\boldsymbol{\beta}$ & 0.53 & 1.08 \\
\hline $\mathrm{F}_{\mathrm{cyl}}$ & 0.1 & 0.1 \\
\hline \multirow{2}{*}{ 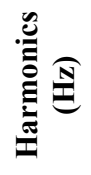 } & $\begin{array}{l}0.2 \\
0.3\end{array}$ & $\begin{array}{l}0.2 \\
0.3 \\
0.4\end{array}$ \\
\hline & 1.5 & $\begin{array}{l}1.7 \\
2.2\end{array}$ \\
\hline
\end{tabular}

\section{CONCLUSIONS}

This paper presents an experimental investigation on oscillatory Couette-Taylor flows. The vortices may manifest themselves by the presence of time- oscillations on mass transfer. The instantaneous and local mass transfer rates evolutions demonstrate the apparition and development of Taylor vortices which manifest themselves by the presence of oscillations having constant amplitudes for $\beta=0.53$ and $\beta=1.08$. The vortices direction can be deduced from the sign of the instantaneous wall shear rate time evolution. The wall shear rate was determined using three approaches: the "Lévêque solution" (1923), Sobolik et al. (1987) and the inverse method (2006). The results illustrate that the modulation can destabilize laminar Couette flow even for low oscillation frequencies and relatively low oscillation amplitudes. Oscillatory flows accelerate the flow transition. The Taylor number of the steady case to obtain the same mean shear rate is greater than the mean Taylor number relative to the oscillatory case. The frequencies spectra allows to characterize the increasingly complex dynamics that emerges with the increasing of $\beta$.

\section{ACKNOWLEDGMENTS}

This work was supported by the laboratory GEPEA (University of Nantes) and the "Agence Nationale de la Recherche (ANR), France", by the grant $n^{\circ}$ ANR08-BLAN-0184-02. These supports are gratefully acknowledged.

\section{REFERENCES}

Abcha, N., Crumeyrolle O., Ezersky A.B., Mutabazi I. (2013) Velocity field of the spiral vortex flow in the Couette-Taylor system, The European Physical Journal E, 36(3):9834.

Barenghi, C. F., Jones C. A. (1989) Modulated TaylorCouette flow", Journal of Fluid Mechanics, 208, 127-160.

Barenghi, C. F. (1991) Computations of transitions and Taylor vortices in temporally modulated Taylor-Couette flow", Journal Of Computational Physics, 95, 175-194.

Berrich, E., Aloui F., Legrand J. (2012a) On the Stability of Taylor Couette Flow With Axial Flow, ASME 2012, FEDSM 2012-72235,899908.

Berrich, E., Aloui F., Legrand J. (2012b) Synchronization between PIV and Electrodiffusion techniques for the characterization of Wavy and Modulated Wavy Taylor Vortex flows with axial flows, Turbulence Heat and Mass Transfer International Conference THMT'12, September 24-28, 2012 (paper code E107), Palermo, Sicily, ITALY, 2012 (b). 
E. B. Betouche et al. /JAFM, Vol. 9, Special Issue 1, pp. 147-154, 2016.

Berrich, E., Aloui F., Legrand J. (2013a) Experimental Study on Oscillatory CouetteTaylor Flows Behaviour, ASME 2013 Fluids Engineering Division Summer Meeting. FEDS2013-16308,

Berrich, E., Aloui F., Legrand J. (2013b) Analysis of Inverse Method Applied on Sandwich Probes, Journal of Fluids Engineering (135) 1,

Berrich, E., Aloui F., Legrand J. (2013c) Experimental validation and critical analysis of inverse method in mass transfer using electrochemical sensor, Experimental Thermal and Fluid Science. 44, 253-263.

Carmi, S., Tustaniwskyj J.I., "Stability of modulated finite-gap cylindrical Couette flow: linear theory", J. Fluid Mech. 108, 19-42, 1981.

Coughlin, K. T., Marcus P. S., (1992) Modulated waves in Taylor-Couette flow Part 1. Analysis, $J$ . Fluid Mech., (234) 1-18, 1992.

Donnelly R. J., Reif F. Suhl H. (1962). Enhancement of hydrodynamic stability by modulation, Physical, Review Letters, 9, 363-365,.

Ganske, A., Gebhardt T., Grossmann S. (1994) Taylor-Couette flow with modulated inner cylinder velocity, Physics Letters A, 192, 75-78.

Gilchrist, S., Ching C.Y. Ewing D. (2005) Heat Transfer Enhancement in Axial Taylor Couette Flow, ASME 2005, HT2005-72746, pp.227-233, ISBN: 0-7918-4731-4,.

Hall, P. (1975) The stability of unsteady cylinder flows", Journal of Fluid Mechanics, 67 (1), 2963, 1975.

Hall P. (1983) On the nonlinear stability of slowly varying time-dependent viscous flows", Journal of Fluid Mechanics, 67 (1), 357-368.

Kedia, R., Hunt M. L., Colonius T. (1999) Transition of chaotic Flow in a Radially Heayed Taylor Couette System, J.Heat Transfer 121(3), 574582.

Kristiawan M., Jirout T., Sobolik V. (2011). Components of wall shear rate in wavy Taylor Couette flow, Experimental Thermal and Fluid Science 35(7):1304.

Lévêque M. A. (1928) Les lois de transmission de la chaleur par convection", Ann. Mines,(13), 381412 .
Lopez J. M., Marques F. (2002) Modulated TaylorCouette flow: Onset of spiral modes", Theor. Comput. Fluid Dyn., 16, 59-68.

Martial Gassa Feugaing, Olivier Crumeyrolle and Innocent Mutabazi (2011) Etude expérimentale de l'écoulement de Couette-Taylor avec modulation de fréquence", Rencontre $d u$ nonlinéaire.

Martinand D., Serre E. and Lueptow R. M., (2009) Absolute and convective instability of cylindrical Couette flow with axial and radial flows, Physics of Fluids, 21104102.

Rehimi F., Aloui F., Ben Nasrallah S., Doubliez L., Legrand J. (2006) Inverse method for electrochemical diagnostics of flows", Int. J. of Heat and Mass Transfer, 49, 1242- 1254.

Riley P .J., Laurence R.L. (1976) Linear stability of modulated circular Couette flow", Journal of Fluid Mechanics, 75 (4), 625-646.

Savaq O. (1985) On flow visualization using reflective flakes", J. Fluid Mech., 152, 235-248.

Skalak R., Ozkaya N. , Skalak T. (1989) Biofluid Mechanics", Annual Reviews Fluid Mechanics, $21,167$.

Sobolik V., Wein O., J. Cermak (1987)Simultaneous measurement of film thickness and wall shear stress in wavy flow of non-Newtonian liquids", Collection Czechoslovak Chem. Comun (52), 913-928.

Swinney H. L., Zhang L. (2013) Taylor Vortex Flow Dynamics, Hands on Research in Complex Systems", School ICTP, Trieste, Italy, 1-12 July

Tilton N., Martinand D., Serre E. and Lueptow R.M., (2010) Pressure-driven radial flow in a TaylorCouette cell, J.Fluid. Mech.

Walsh T. J., Donnelly R. J. (1988) Stability of modulated Couette flow", Physical Review Letters, 58, 2543-2546.

Youd A. J., Willis A. P., Barenghi C.F. (2003) Reversing and non-reversing modulated TaylorCouette flow"', J. Fluid Mech., 487, 367-376.

Youd A. J., Willis A. P., Barenghi C. F. (2005) Nonreversing modulated Taylor-Couette flows", Fluid Dynamics Research, 36, 61-73,

Zhang L. H, Swinney H. L. (1985) Nonpropagating oscillatory modes in Couette-Taylor flow", Physical Revue A 32 (2). 
E. B. Betouche et al. /JAFM, Vol. 9, Special Issue 1, pp. 147-154, 2016. 